\title{
Tools, Tricks, and Hacks: Exploring Novel Digital Fabrication Workflows on \#PlotterTwitter
}

\author{
Hannah Twigg-Smith \\ htwigg@uw.edu \\ University of Washington \\ Seattle, Washington, USA
}

\author{
Jasper Tran O’Leary \\ jaspero@uw.edu \\ University of Washington \\ Seattle, Washington, USA
}

\author{
Nadya Peek \\ nadya@uw.edu \\ University of Washington \\ Seattle, Washington, USA
}

\begin{abstract}
As digital fabrication machines become widespread, online communities have provided space for diverse practitioners to share their work, troubleshoot, and socialize. These communities pioneer increasingly novel fabrication workflows, and it is critical that we understand and conceptualize these workflows beyond traditional manufacturing models. To this end, we conduct a qualitative study of \#PlotterTwitter, an online community developing custom hardware and software tools to create artwork with computer-controlled drawing machines known as plotters. We documented and analyzed emergent themes where the traditional interpretation of digital fabrication workflows fails to capture important nuances and nascent directions. We find that \#PlotterTwitter makers champion creative exploration of interwoven digital and physical materials over a predictable series of steps. We discuss how this challenges longrunning views of digital fabrication and propose design implications for future frameworks and toolkits to account for this breadth of practice.
\end{abstract}

\section{CCS CONCEPTS}

- Human-centered computing $\rightarrow \mathrm{HCI}$ theory, concepts and models.

\section{KEYWORDS}

fabrication, maker culture

\section{ACM Reference Format:}

Hannah Twigg-Smith, Jasper Tran O’Leary, and Nadya Peek. 2021. Tools, Tricks, and Hacks: Exploring Novel Digital Fabrication Workflows on \#PlotterTwitter. In CHI Conference on Human Factors in Computing Systems (CHI '21), May 8-13, 2021, Yokohama, Japan. ACM, New York, NY, USA, 15 pages. https://doi.org/10.1145/3411764.3445653

\section{INTRODUCTION}

As computer-controlled digital fabrication machines become more affordable, they become increasingly accessible to a diverse user base. Online hobbyist communities have cropped up around digital fabrication machines, comprising people who use and customize both the machines and their supporting software tools [2, 48, 68].

Permission to make digital or hard copies of all or part of this work for personal or classroom use is granted without fee provided that copies are not made or distributed for profit or commercial advantage and that copies bear this notice and the full citation on the first page. Copyrights for components of this work owned by others than the author(s) must be honored. Abstracting with credit is permitted. To copy otherwise, or republish, to post on servers or to redistribute to lists, requires prior specific permission and/or a fee. Request permissions from permissions@acm.org.

CHI '21, May 8-13, 2021, Yokohama, Japan

(C) 2021 Copyright held by the owner/author(s). Publication rights licensed to ACM. ACM ISBN 978-1-4503-8096-6/21/05 ...\$15.00

https://doi.org/10.1145/3411764.3445653
These organic communities, made up of people with various backgrounds and levels of expertise, also create and share bespoke digital fabrication workflows. We define a workflow as the series of steps a person takes to move from design concept to final product, often consisting of various software tools, file formats, machine code and behavior, and material forms.

Much of the software and hardware infrastructure underlying digital fabrication tools today was developed for engineering settings in the mid-twentieth century. This infrastructure prioritizes standardization and repeatability for expert users, which has led to a system of normative ideas of what constitutes engineering as distinct from art and craft. Over time, this has resulted in a prescriptive stance of how digital fabrication should be used even beyond traditional engineering environments, replete with assumptions about who the users of the technology might be.

In contrast to traditional engineering environments, personal digital fabrication machines allow for highly customized, smallscale manufacturing. In this paper, we explore an online community, \#PlotterTwitter, which challenges normative descriptions of digital fabrication. Instead of exclusively working within existing prescriptive workflows, members of \#PlotterTwitter claim the agency to develop personal, ad hoc software tools that support their individual artistic goals. Furthermore, critically examining where individuals are creating their own software and hardware tools would allow HCI researchers to understand how makers in online communities are pushing the boundaries of what can be produced with these tools.

Yet, many HCI researchers and practitioners continue to theorize the whole of digital fabrication as a canonical workflow that proceeds rigidly through several consecutive stages: a design intent, a digital representation, machine instructions, and a final product, as schematically shown in Figure 1 . This is what is heralded as the future in which "data becomes things" [20]. However, the data we gathered from online fabrication communities show us that the range of workflows that members are pioneering extends far beyond what can be captured in this common-case canonical workflow. As previous "edge cases" become the new status quo, it is more important than ever to develop models of digital fabrication workflows that account for people who are building their own infrastructure for creative goals.

To examine how online digital fabrication communities are working beyond the canonical workflow, we conducted a qualitative case study of \#PlotterTwitter, a community of Twitter users who document and publish their artistic work under the eponymous hashtag. Members of \#PlotterTwitter use plotters, computer-controlled drawing machines that move a pen or other writing instrument to produce a 2D drawing. Members share final drawings that result from 


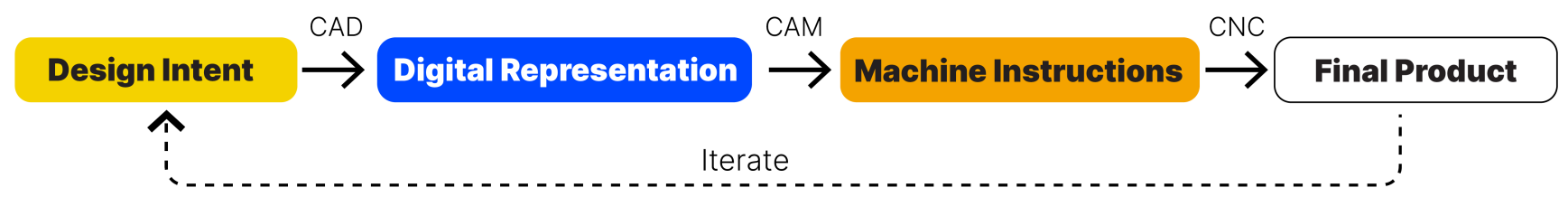

Figure 1: The canonical digital fabrication workflow implies a linear progression through CAD, CAM, and CNC.

their workflows, but also share works-in-progress, screenshots and code from software tools, photos of machine use and machine failures, and snippets from bespoke workflows-in-progress. We chose to study \#PlotterTwitter in particular due to the low barrier to entry to plotters compared to that of other digital fabrication machines. Furthermore, participants arrive at \#PlotterTwitter from a wide range of backgrounds outside of maker communities, such as craft and illustration. As a result, \#PlotterTwitter offers a diverse sample of community members of varying levels of expertise who document their activities frequently and publicly. While the medium of Twitter introduces some constraints, such as the brevity of tweets compared to longer-format tutorials or forums, \#PlotterTwitter remains an evolving collection of workflows with both a low barrier to machine use and a low barrier to posting content and engaging in discussion.

Given our goal of better understanding the diverse and social development of plotter workflows, we propose the following research questions: How can we conceptualize the space of workflows on \#PlotterTwitter beyond the canonical workflow? and How does work from \#PlotterTwitter question assumptions of who uses digital fabrication tools? While both questions are specific to \#PlotterTwitter, they are also useful for studying communities where members are actively developing and sharing new ways of engaging with digital fabrication machines.

To address these questions, we conducted an analysis of 1500 tweets from \#PlotterTwitter that were collected over five months. To guide our analysis, we created a set of codes corresponding to all possible transitions between steps in the canonical workflow. These codes are not intended to fully capture the range of workflows on \#PlotterTwitter-instead, they represent the extent to which we can conceptualize workflows using the canonical workflow as a theoretical frame. They also give us a starting point for discussing the niche and personalized processes of personal fabrication. To this end, while coding tweets, we paid particular attention to approaches that were were poorly captured (or not at all captured) by this new model. We created memos of serendipitous observations and used them to identify and develop themes. We detail and discuss each theme, and use them to argue for the necessity of a more inclusive understanding of the breadth of workflows in digital fabrication.

\section{BACKGROUND}

\subsection{Machine Histories, Social Resistance, and Standardization}

Demands for metal products during the Second World War intensified development of new machines and technology for making these products. The invention of computer-numerically-controlled (CNC) machinery was driven by a need for efficiently produced complex parts. However, beyond efficiency, developing CNC also provided an opportunity to reshape power relations between machinist labor and management [47]. The technology that grew into $\mathrm{CAD} / \mathrm{CAM}$ (computer-aided design/computer-aided manufacturing) was partially shaped by a desire to reduce reliance on skilled labor. Stakeholders at the time argued that the separation of programming CNC machines (complex, happens once) from running CNC machines (once coded, can easily be repeated) could facilitate deskilling manufacturing and reducing production cost. Production could scale up with an investment in machines, rather than people.

This separation in programming and running machines persists in contemporary digital fabrication in a canonical workflow where steps can be categorized as CAD - CAM - CNC (see Figure 1). These steps separate an object's design, its manufacturing plan, and its manufacturing process. This categorization of steps is familiar to the HCI community-for example, in describing the stages newcomers go through when 3D printing, Hudson et al. name 1. Plan, 2. Model, 3. Setup, 4. Verify, 5. Print [26]. They found that the expertise required for $3 \mathrm{D}$ modelling in $\mathrm{CAD}$ was a major barrier to newcomers, and thus many novices trying out 3D printing turned to websites such as Thingiverse in search of premade designssubstituting downloading for modelling. However, Hudson et al., a more in-depth analysis of activity on Thingiverse [3], and a study of digital fabrication practitioners [81] show that design decisions made in CAD are tightly coupled to viable choices in CAM and $\mathrm{CNC}$. They highlight that successful digital fabrication relies on contextual information, design clarification, and expert tips that inform other stages of making.

In Forces of Production, Noble, through extensive anthropological research, describes how although companies adopting CNC equipment in the 1960s argued the CNC was easier to operate and therefore should be compensated at lower rates, labor tasked with operating the equipment found its operation to be error-prone, to require greater attention and anticipation to avoid collisions and problems, and to cause more worker tension and fatigue. To effectively run the machines, workers relied on skill and experience to know when to pause operation, make adjustments, or check tolerances. Protracted conflict with labor eventually resulted in companies conceding that operating $\mathrm{CNC}$ equipment needed to be done at a rank equal to if not higher than those operating conventional equipment [47, Chapter 11]. HCI and STS scholars have a long-standing tradition of studying such disconnects between representations of work and practices of work including in laboratory studies [33, 36, 63], entrepreneurship [21, 28, 38], and medicine 
$[15,44]$. We argue that digital fabrication workflows would benefit from further scrutiny distinguishing between promise and practice and position our contribution in this tradition.

HCI fabrication research contains many systems contributions, for example providing proof-of-concept implementations of novel workflows [61, 83]. Many of these contributions are not made for existing communities of practice (such as machinists or craftspeople), but for "novices", "consumers", or "non-engineers" who are predicted to engage more with digital fabrication in the near future if related technology is made increasingly available and accessible. To underline how non-experts could engage with fabrication through these systems, many are described as "end-to-end", or that the system encompasses all steps seamlessly and no further workflow development or problem solving is required from the user. Examples of end-to-end fabrication systems for novices include those for designing and making laser-cut objects [7], pneumatic devices [40], bottle-based assemblies [34], laminated mechanisms [37], knit objects [46], button pressing systems [58], origami robots [42], or molds for casting [78]. Each of these systems offers a clear pathway for design and fabrication within a particular workflow, but not necessarily a clear picture for who might adopt those workflows and why.

Suchman contends that human actions are always situated and contextual [72], which erodes the value of pre-determined plans. We believe this erosion applies to many seamless fabrication systems. On \#PlotterTwitter, we observe extensive resistance to the prescriptive plan of the canonical workflow. This is despite the breadth of backgrounds, novice or otherwise, present in the \#PlotterTwitter community. We argue that the categories of the canonical workflow are too constrictive to describe \#PlotterTwitter's digital fabrication practice. Furthermore, these categories have origins in a system designed for control, rather than agency. We identify a need to understand how people practice digital fabrication in context, such that we can approach the design of digital fabrication technology while keeping human agency in the center of our focus.

\subsection{Craft and Computation}

Scholars have already explored the intersection of craft and computation through various angles. We are most interested in expanding on work that asks: what happens when machines become co-creators of craft, rather than static automators? Andersen and colleagues examine this notion explicitly with their notion of digital crafts-machine-ship, writing that "in this dialogue of closeness and distance, we are still making and changing the machine, and what the machine makes. This is live coding or live building: we touch the system and the system touches us back" [4].

The idea that the maker is affected by what they are working with-from materials, to machines and tools, and now to the software that controls machines-is what social anthropologist Ingold terms morphogenetic design [27]. Morphogenetic design contrasts with hylomorphic design, inherent to the canonical workflow, which positions the maker as the single source of design intent and machines as fixed implementors. He writes that "whenever we read that in the making of artefacts, practitioners impose forms internal to the mind onto a material world 'out there,' hylomorphism is at work." Driven to imagine digital fabrication outside of hylomorphism, Devendorf et al. propose the notion of postanthropocentric making where the maker "becomes innovative and technologically fluent by working closely with machines and materials, listening and sensing their actions, and responding accordingly" [13]. They and other scholars have proposed systems that highlight the agency of the material, from the the materiality of light [70,75], to hybrid smocking [14]. Of particular note is FreeD, a hybrid carving tool that incorporates both the manual action of the maker alongside the data from a 3D model [83]. More recently, Albaugh et al. built an "undeterministic" loom where the final result is influenced by the actions of the weaver, sampled camera imagery, and community participation via the Twitch streaming platform [1]. While these works show curated instances of morphogenetic crafting with machines, we are interested in how morphogenetic craft is happening within \#PlotterTwitter. We seek to understand where makers at a grassroots level organically depart from hylomorphic notions of digital fabrication.

Conceptions of the machine as a co-creator led us to explore the relationship between \#PlotterTwitter artists and their self-made software tools. Software tools for generating vector graphics, particularly prominent on \#PlotterTwitter, are ephemeral and distinct from the physical tools that craft literature has often focused on. They occupy an intermediary place in the creators workflow, generating a plan for what is to be drawn without drawing it. These tools, and the abstractions required to create them, have the effect of separating the creator from their materials [30]. Of physical ad hoc tools in hackerspaces, Bardzell et al. write: "The tool not only clarifies one's work but also situates one in relation to the world, understood in both physical and also social senses." [6]. Wakkary and Maestri further reinforce this idea through their ethnographic study of resourceful design in the home. [79] Now, how might this sentiment evolve when applied to software tools for digital fabrication?

We also draw inspiration from HCI scholars who draw from theories of craft more generally. Blauvelt et al. examine how computational craft re-informs interpretations of hardware and software [8], and Rosner calls attention to the role of craft within the development of computing technology, and its implications for the field of design [65]. Frankjaer and Dalsgaard contribute a review of craft-based inquiry in HCI leveraging Sennett's tripartite model of craft $[18,71]$. We can observe their notions of "creating knowledge through deep, embodied engagement" in the work of various artists working at the edges of digital fabrication $[43,60,66]$. Scholars have leveraged paper in particular, from circuit stickers [56], animation [55], kirigami input devices [11, 82], foldable mechatronics [49, 69], to machines themselves [53]. Other researchers have explored ways of bringing craft-based thinking into computational mediums, notably, Jacobs's Dynamic Brushes [29], He and Adar's embroidering plotter [22], and Tian et al.'s improvisational woodworking tool [74]. Prior work has also examined how digital fabrication tools are gaining traction in settings where they have not previously existed. Quitmeyer and Perner-Wilson contributed the notion of digital craft in harsh environments [57], while Jacobs and Zoran explored combining the traditional crafting techniques of Kalahari hunter-gatherers with computational tools [31]. Saegusa et al. and Devendorf et al. have explored how digital fabrication tools disrupt or integrate with traditional craft workshop settings in a pottery studio and a weaving workshop, respectively $[12,67]$. 


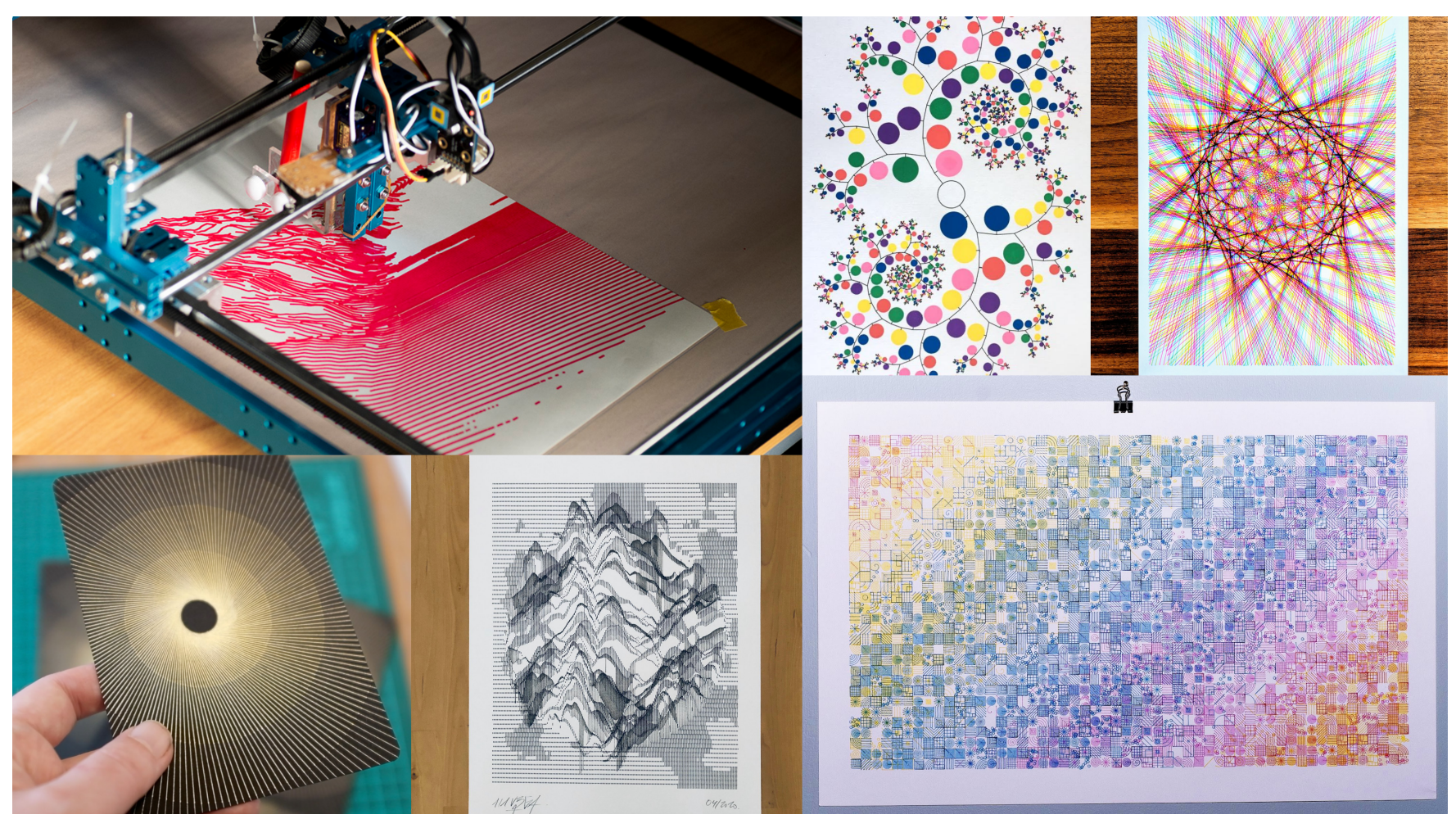

Figure 2: \#PlotterTwitter images shared on Twitter by @NadiehBremer, @v3ga, @jcupitt65, @paulrickards, @revdancatt, and @kenjirototsuji.

Overall, the coupling of digital fabrication and craft is an active area, and prior work has investigated the intersection in many domains. This paper, while relevant to this body of work, differs in that we are focusing on studying makers who are are engaging with craft and digital fabrication in online communities. We investigate how people are not only redefining craft through computation, but redefining computation as part of their craft on \#PlotterTwitter.

\subsection{Online Creative Communities}

Prior scholarship of online creative communities has proliferated, covering a vast range of domains. We are most interested in work that explores themes found in \#PlotterTwitter such as do-it-yourself (DIY) ethos, peer production, and remix culture. One thread examines the Scratch programming language and its associated community, which has expanded far beyond its initial goal of promoting children's learning. Studies of this community examine trends in creative collaboration, [64], remixing [24], and attribution [45]. Programming languages dedicated to creative coding, such as Processing, p5.js, and openFrameworks, have enabled people to create art with code. Communities emerge from each language's respective forum [50,54], often contributing back to the design of the language [51].

More specifically, researchers have studied how entire processes are documented and shared across the internet. Kusnetsov and Paulos highlight online DIY communities such as Instructables, Adafruit, and Etsy [35], finding that such communities emphasize open sharing and learning over profit and social capital. Tseng and
Resnick study Instructables in-depth, analyzing how creators document their projects [77]. Other scholars have studied online fan fiction communities $[5,16]$. In particular, Kim et al. contribute a prototype tool to allow small online groups to crowdsource writing short stories [32]. Some of these online communities, such as the World of Warcraft machinima community, develop their own tools in tandem with establishing a community aesthetic and visual skill [52]. Digital fabrication has its share of online communities that afford remixing physical models [48] which have inspired novel systems that allow creatively reconfiguring mesh files [76] and linking 3D objects [25]. Our interest lies in identifying how lessons from prior work carry over to \#PlotterTwitter, where the the underlying software and machine infrastructure become part of the craft itself.

\section{METHODS}

During ongoing exploration of online maker communities, we identified \#PlotterTwitter as a place where examples of diverse practice are shared and iterated upon by a wide audience. We are interested in documenting the variety of techniques people use to create their plotted artwork. To analyze workflows on \#PlotterTwitter, we developed a coding scheme to describe workflow steps. We collected data from \#PlotterTwitter using Twitter's streaming API, and three coders assigned codes to the data using a custom browserbased tool. We combined the coded data and documented emergent themes, including where our new coding scheme failed to capture the nuances of individual workflows. 


\subsection{Identifying Twitter Accounts}

We have decided not to anonymize the Twitter handles of the \#PlotterTwitter artists whose works are included in this paper. We have contacted or attempted to contact all 29 accounts that appear. All of those who have responded (25/29) have asked for their Twitter handles to be included alongside their work, mentioning that they use the hashtag for recognition and exposure. Given a clear community preference for named attribution, we believe it is still appropriate to credit the remaining four Twitter accounts for their creative work, even though we were not able to secure their explicit consent (see [9]). It is also worth noting that the four accounts we were unable to contact were seemingly anonymous and not linked to identifying information such as human names, personal websites, or other social media. We have also added all 29 accounts to the paper's acknowledgements section.

\subsection{Background on Plotter Machines and Plotter Software}

Plotters are $\mathrm{CNC}$ machines that draw lines based on vector graphics. They were one of the earliest output devices available to computers, first appearing in the 1950s. Historically, they also had an advantage over conventional printers for producing large technical drawings, and were widely used in architecture and engineering. Vector graphics (e.g. SVG files) describe shapes as mathematical functions rather than by listing values of individual pixels. These functions include a direction, start, and end point, and are rendered on-screen as a line or smooth curve. However, plotters ultimately deal with movement, accelerating, decelerating, and lifting the pen at different speeds. This information is invisible when viewing a vector drawing alone on a screen. Paired with the variable properties of paper, ink, and other materials, the translation from screen to physical product is far from "push-to-print." Plotters are no longer widely used in industry, but they have been adopted by craft and hacker communities. Popular brands include Axidraw, Cricut, and Silhouette. To convert from vector file formats to plotter motion, the vendors provide control software, similar to a printer driver, that assists the users with creating a toolpath.

\subsection{Details of \#PlotterTwitter and Related Online Communities}

The \#PlotterTwitter hashtag is actively used on social media platforms including both Twitter and Instagram to share content relating to the use of plotters, usually for the creation of art. Figures 2 and 6 contain example images from our dataset that we consider to be representative of the work people share on \#PlotterTwitter. Other communities devoted to drawing machine discussion have emerged outside of Twitter, including dedicated groups on Facebook, Discord, and Reddit. The most active of these other communities seems to be the DrawingBots Discord server, which had approximately 800 members in January 2021. In addition to sharing completed and in-progress works, members of these communities ask questions, share resources and ideas, and engage in other general discussion related to plotters and drawing machines. In early 2019, a member of the community created drawingbots.net, a website for compiling resources for plotting, such as vector graphics generators, pen and paper recommendations, and numerous custom software tools for eliciting unconventional behavior from their plotters. Since 2018, the Discord community has organized a number of in-person meetups and artwork swaps in London, New York City, and San Francisco.

\subsection{Codebook}

Through initial grounded observations, we noticed that the canonical CAD/CAM/CNC workflow often used to frame HCI fabrication research was not able to capture the breadth of \#PlotterTwitter workflows. We found that artists often shared tools that they had made themselves, or described activities that could not be accurately described as CAD, CAM, or CNC. Using the canonical workflow as a starting point, we connected the nodes of the workflow graph in Figure 1, naming additional edges between categories of the canonical workflow, describing practices that cannot be directly categorized as CAD, CAM, or CNC. We arrived at ten total codes for describing workflow steps, which are shown in Figure 3. We name and describe each workflow step code, and provide a series of example tweets.

CAD: Computer-Aided Design. The term "CAD" is often used in engineering circles to refer to design software, often (but not exclusively) used for defining geometry. We are using "CAD" to describe any task that translates a user's design intent to a digital representation. Some examples of CAD software include 3D modeling programs like Blender and Rhino3D, and 2D vector graphics programs like Inkscape and Adobe Illustrator. For our analysis we also include self-developed tools in this category, like ones often used in creative computation such as Processing scripts or browserbased SVG generators. In an example CAD tweet, Twitter user @clarkkampfe shares a recent CAD tool they created (Figure 4a): "My latest. You can make your own with the tool I recently published at https://rasterize.live \#plottertwitter Make sure to select the option to output SVG."

CAP: Computer Aided Planning. This workflow step fulfills a similar place as the task colloquially known as CAM (Computer-aided Machining/Manufacturing). However, we opted to use the more generic term "planning" to better describe this step, as automation is not limited to machining or manufacturing. During the CAP step, a digital representation of a task or object is converted into machine instructions (in a language such as G-code) that can be executed on a machine. Software that provides this functionality includes Cura (for 3D-printing), or homebrew SVG-to-G-code conversion tools. In an example CAP tweet, Twitter user @lwrkalia asks for advice on converting SVGs to G-code: "What do \#plottertwitter people use for converting SVGs to g-code? Looking for something that can be automated." After receiving some responses, they find a process that works: "Right now I take my SVGs, then process with vpype then generate gcode with juicy gcode. Very happy with the results."

MEX: Machine Execution. During machine execution, a set of lowlevel machine instructions, such as G-code, is executed by a physical machine. In one tweet, @ManiacalLabs shares a video of their plotter plotting its own schematic, revealing G-code instructions that were generated with Lightburn, a program for controlling laser cutters. 
DMC: 260

\begin{tabular}{lll} 
Activity Key: & & \\
& & \\
Computer-Mediated Automation & CMA: & 0 \\
Intent Iteration & INI: & 10 \\
Direct Execution & DEX: & 12 \\
Plan Iteration & PLI: & 15 \\
Physical Prototype Iteration & PPI: & 21 \\
Computer-Aided Planning & CAP: & 23 \\
Digital Representation Iteration & DRI: & 66 \\
Computer-Aided Design & CAD: & 236 \\
Direct Machine Control & DMX: & 260 \\
Machine Execution & MEX: & 643 \\
& OTHER: & 776 \\
\hline & TOTAL: & 1506
\end{tabular}

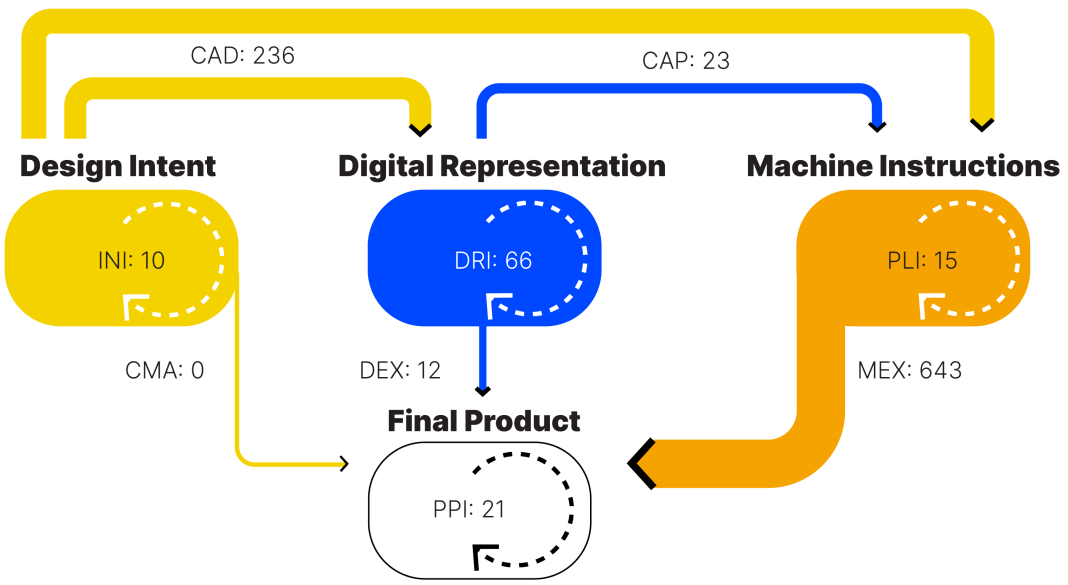

Figure 3: A list of our codes and their occurrences. Our codes draw additional connections between categories of the canonical workflow, describing practices that cannot be directly categorized as CAD, CAM, or CNC.

DEX: Direct Execution. During direct execution, a separate set of machine instructions is not explicitly created. Instead, the machine is controlled directly from the digital representation. For example, the Axidraw Control plugin for Inkscape allows a user to directly control the Axidraw without exporting an SVG and converting it to G-code. An example DEX tweet by @mhkingsbury uses this plugin to draw a map (Figure 5b): "I used @anvaka's City Roads to build the map of @stlouispark and sent it to the AxiDraw for printing. Really happy with how it turned out. \#PlotterTwitter."

CMA: Computer-Mediated Automation. Computer-mediated automation includes systems where a machine immediately translates a user's physical input into a physical result. This code is similar to DEX, however, instead of a completed set of machine instructions being run on a machine, individual commands are sent to the machine as a user makes decisions. While we did not find any examples of CMA in our dataset, systems dubbed as interactive fabrication [80] in HCI literature, such as Tian et al.'s Turn-by-Wire, would be considered computer-mediated automation. [73].

INI: Intent Iteration. Intent iteration represents activities that help refine the user's intent. This could include brainstorming, material explorations, sketches, or other processes. Intent iteration aids in solidifying future workflow steps by forming a more-complete end goal.

DRI: Digital Representation Iteration. Digital representation iteration describes iterating on existing digital representations. For example, someone could generate artwork in Processing, export it as an SVG, and use Inkscape tools to remove curves that they do not want to appear in the final artwork. In an example DRI tweet, @fastness describes an algorithm they created to convert digital photos to vector art: "First draft of a photo to line drawing algorithm I've been thinking about for years, on and off. The bits where it goes wrong are sort of interesting at least... \#plottertwitter."

PLI: Plan Iteration. Plan iteration describes tasks that iterate on a set of machine instructions. This could include compilation, path optimization, or validation. In an example PLI tweet, @thomasp85 shows the results of their path optimization tool (Figure 4b): " $M y$ \#axidraw graphic device now has automatic travel optimisation as well as a preview rendering mode. Red lines are air travel \#plottertwitter \#rstats."

PPI: Physical Product Iteration. This workflow step describes activities which take place by a human, often after a machine has been used. This includes tasks such as sanding, painting, or other finishing techniques. PPI could also describe human tasks that take place in parallel with the machine execution. In an example PPI tweet shown in Figure 4c, @265design shares a workflow where they use a plotted design to cut and fold paper by hand: "Digital to $2 D$ to $3 D$ \#paperart \#paperfolding \#penplotter \#plottertwitter \#geometricart.”

DMC: Direct Machine Control. The direct machine control workflow step represents activities that bypass the creation of a digital representation. This could include manually writing lines of G-code or using machines that allow for record-and-playback functionality. We also consider machine setup activities such as zeroing or calibrating pen heights to fall into the DMC category, as the user generally directly enters commands to move the machine. A DMC example tweet by @plotslol demonstrates a calibration tool they created for their plotter (Figure 5a): “my hobby isn't making art with a plotter, it's making artful calibration tools FOR my plotter \#PlotterTwitter."

OTHER. We use the "OTHER" code to describe tweets that could not be described the previously discussed workflow codes. This included tweets that were off-topic or participating in \#PlotterTwitter meta-discussion: "I discovered \#PlotterTwitter today and my life has been changed" - @mimigenot However, the "OTHER" code also allowed us to see where our coding scheme fell short of describing all of the activities on \#PlotterTwitter. We found that many seemingly important workflow steps ended up included in the OTHER category, as they didn't fit with any of the previous categories. We took note of these additional workflow steps because they could guide 


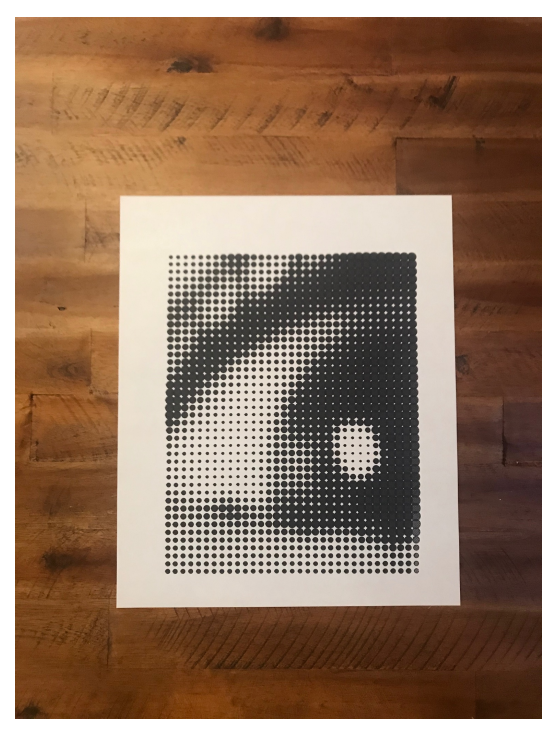

(a) Code CAD: A drawing made by @ clarkkampfe with a custom CAD tool.
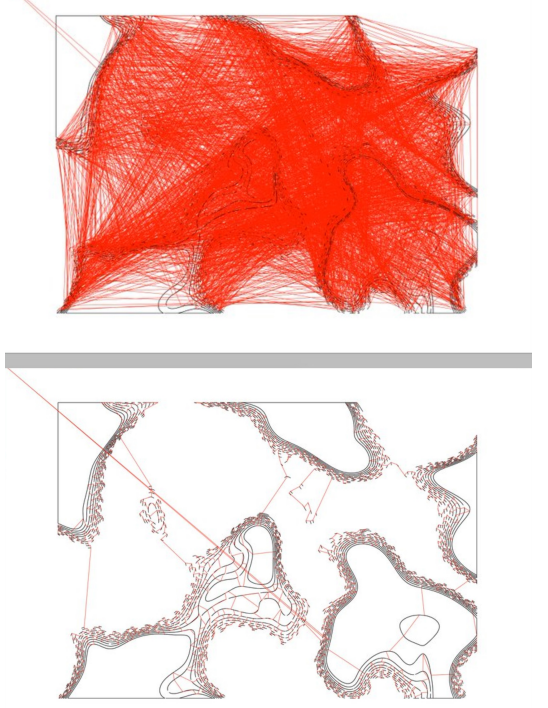

(b) Code CAP: Top: Unoptimized toolpath (pen-up movement in red). Bottom: Optimized toolpath.Via@thomasp85.

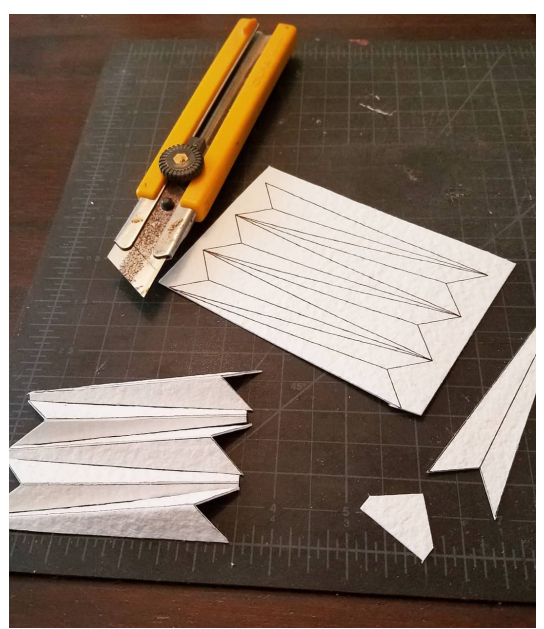

(c) Code PPI: @265design cuts and folds a plot.

Figure 4: The \#PlotterTwitter community shares their work at many points in their processes, not only the finished product.

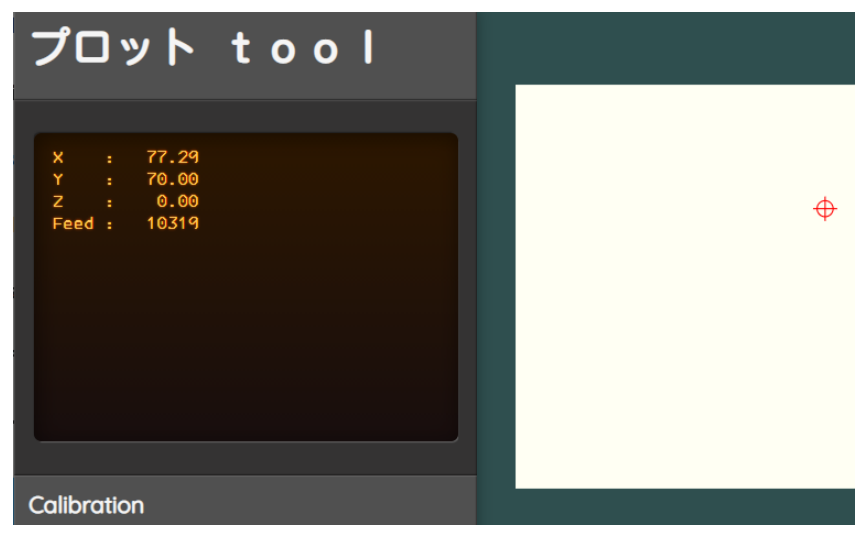

(a) Code DMC: A plotter calibration tool by @plotslol.

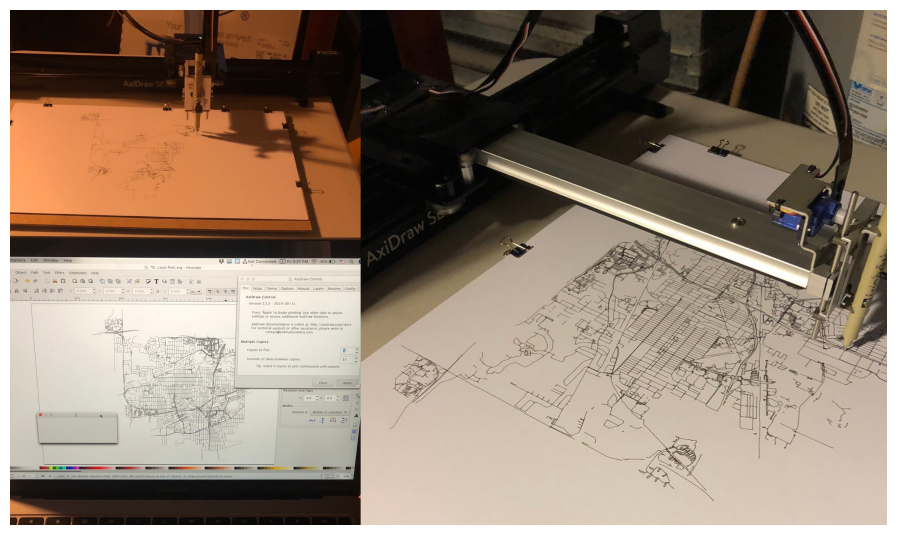

(b) Code DEX: @mhkingsbury controls an Axidraw with the Axidraw Control plugin for Inkscape.

Figure 5: In addition to artworks, people on \#PlotterTwitter also shared the tools they used for calibrating and controlling their plotters.

development of a future workflow framework. One such example was when @liviopacifico used a 3D scanner to transfer real-world geometry to digital form to create a layered ribbon portrait of their sister (Figure 6, right).

\subsection{Data Collection}

Our dataset consisted of 1500 tweets collected between 11 March 2020 and 9 July 2020. We used the Twitter Streaming API to collect tweets over this period, filtering them based on the \#PlotterTwitter hashtag. We ran two separate instances of the streaming API on Heroku virtual servers to prevent possible data loss. Once the data had been collected, we ensured each tweet was represented only once by removing retweets, duplicates of the same tweet ID, and tweets from Plotter Bot (@PlotterTweeter), a bot that shares \#PlotterTwitter content.

\subsection{Coding and Memoing Process}

Three coders individually assigned codes to the entirety of the initial dataset and the results were combined such that one tweet would receive all codes that any coder assigned to it. Because tweets 
could mention multiple steps of a workflow, they could be assigned more than one code. We developed a web application to allow us to efficiently process the tweets. The app loads a JSON file of Twitter data and displays the tweet text and images alongside the code options. The coders could quickly navigate the dataset and assign codes via the keyboard.

The coders set aside any tweets that were coded as OTHER that they felt were serendipitous discoveries. Rivoal and Salazar define serendipity as the "convenient notion to point out the very experience of suddenly coming to 'see' something that had previously been out of sight: the striking realisation that 'this is what it is about' [which] ethnographic narratives refer to as the 'Geertzian moment"' $[19,62]$. The OTHER code intentionally represents both unrelated and non-descriptive tweets, but also highly descriptive tweets that could not fit any of our codes derived from the canonical workflow. The latter revealed unexpected "happy accidents" such as exploiting the inconsistencies in a plotter, or creating from scratch the missing "æ" glyph for a plotting typeface. The coders and authors collectively memoed and discussed these tweets, and the resulting emergent themes inform the majority of our findings.

\subsection{Limitations}

Interest in drawing machines extends beyond the \#PlotterTwitter hashtag. Discord, Reddit, Facebook, Instagram, and other online forums host communities adjacent to \#PlotterTwitter. Interviews with members of these communities could shed light on individual workflow patterns, as the 280-character limit imposed on tweets may prevent \#PlotterTwitter users from sharing nuanced information about their workflows. By coincidence, data collection for this project began around the same time as stay-at-home orders prompted by the COVID-19 pandemic were imposed around the world. We did not compare our data to a pre-pandemic dataset.

\section{FINDINGS}

The workflows displayed on \#PlotterTwitter are incredibly diverse. Artists create their own tools for generating graphics and controlling their machines, and fluidly adopt existing tools as well. In this section, we provide a broad overview of the \#PlotterTwitter content and describe the themes we observed in our dataset. We would like to emphasize we are unable to make claims about individual practice based on the tweets we have analyzed, as we are essentially seeing out-of-context snapshots of individual workflows. Rather, and to the purposes of our argument, the data shows the community's diversity by demonstrating the sheer breadth of practice that takes place on \#PlotterTwitter.

\subsection{Data Overview}

The \#PlotterTwitter hashtag encompasses a range of content, including images of final works and works-in-progress, questions and recommendations, and meta-comments. Figure 2 shows example images of artwork posted with the \#PlotterTwitter hashtag. Most of the pieces we observed on \#PlotterTwitter were abstract, generative, or math-based vector images. However, we also saw artworks where other formats were converted into something plottable, such as paintings, photographs, or 3D-scans. Examples of these pieces are shown in Figure 6.
In the brief 280-character span of a tweet, people who posted artwork generally shared the name of their piece along with the mediums used to create it, similar to how art is traditionally displayed in a gallery. Nearly all the tweets that posted original plots also named the machine they used-Axidraw, Makeblock, and Sillhouette are popular plotter brands we observed. For this reason, the MEX code was well-represented in our dataset, with 643 tweets. Less frequently, the tweet would also name the software tools they had used for some or all of their workflow. This was demonstrated by fewer occurrences of the codes CAD, CAP, DRI, and PLI. Machine instructions, while vital to plotting, were seldom mentioned. We suspect the artists on \#PlotterTwitter generally use tools that could be coded as CAD, CAP, DRI, or PLI, but they do not frequently share them in their tweets. We also saw many cases where elaboration about tools and workflow followed the initial tweet in a thread, or was prompted by a question from a Twitter follower.

\subsection{An Engaged Community Cultivates a Diverse Body of Knowledge}

Our data revealed a vibrant community where \#PlotterTwitter artists share plots, knowledge, and resources. They frequently recommended off-Twitter, community-created resources to each other, such as drawingbots.net or the Drawing Bots Discord server. This community relationship also extended into the physical world. During our data collection, some of the \#PlotterTwitter community organized an artwork swap where they exchanged plots by mail. Other artists occasionally sent out a series of postcards with plots on them, such as @paulrickards (Figure 7).

We observed people on \#PlotterTwitter constantly asking for recommendations for tools to use during their workflows. These questions ranged across a variety of topics, such as finding a consistent way to calibrate pen height, optimizing speed and timing options, converting vector artwork to G-code, tips and tricks for generative art, and many more. Some questions were very specific, such as one from @cicerothedoge: "Dear \#PlotterTwitter I forgot something before ordering my \#axidraw... I'm in Denmark, where

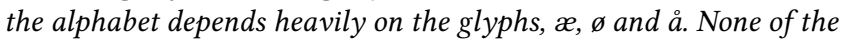
Hershey fonts have all 3, so I will have to create my own. Can anyone recommend a single stroke font creator for Win10?" Other questions were broader: "\#PlotterTwitter what software do you use for generating your beautiful paths? What are the cool programs/methods you know of? Are there any that can fill an area of an SVG with a pattern like a Gosper curve? What about ones for converting raster images? Teach me your ways!"

These tweets reveal that \#PlotterTwitter members frequently tap into the collective body of knowledge maintained by the community. This was particularly true regarding workflow minutia such as wrangling file formats. These details, which could be overlooked in scenarios like a video tutorial or instructional blog post, were often the focus of conversation. We found many instances of questions about software tasks such as generating and exporting SVGs, converting various image formats to G-code, and what machine control software was best for a certain situation. We also observed questions about machine hardware and physical properties: which plotter to purchase or build, how to weight pens to achieve a cleaner line, or how to create adapters for a specific pen. 


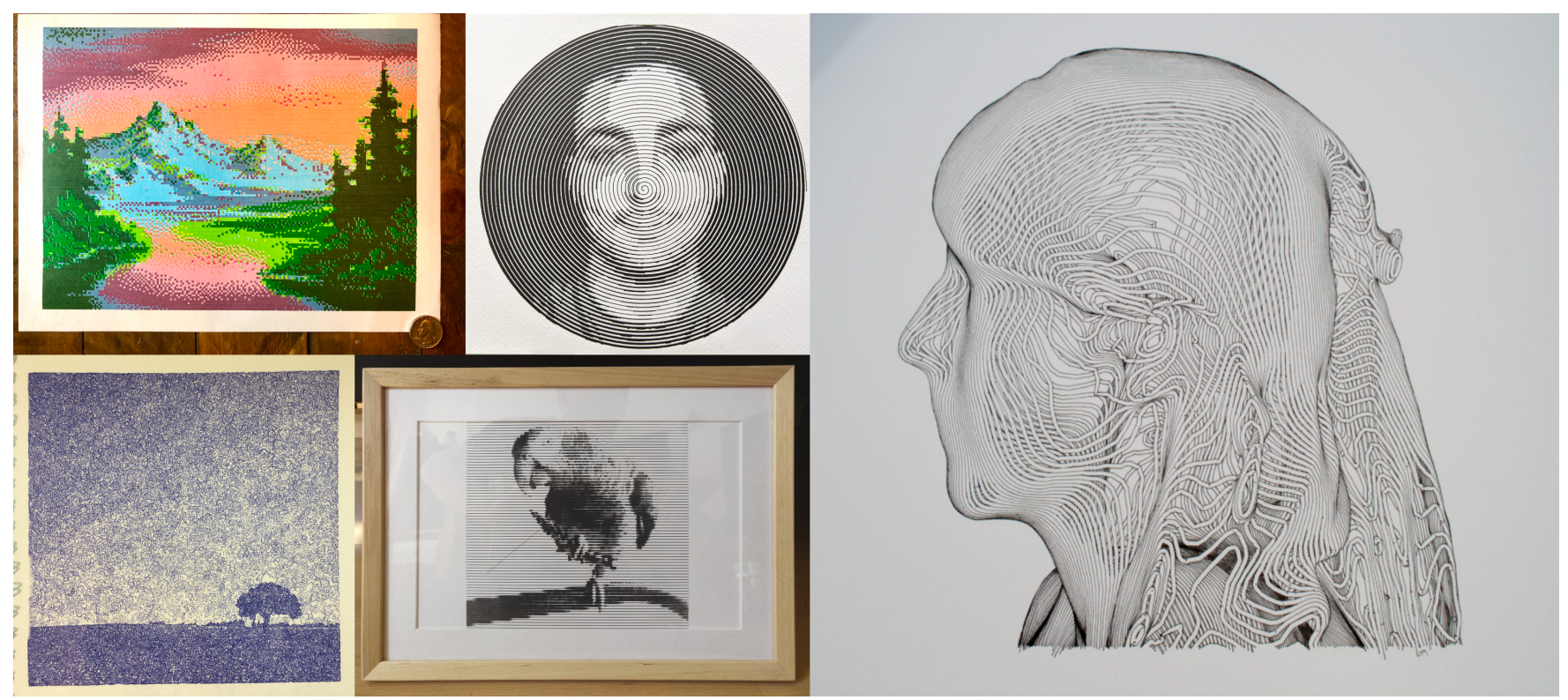

Figure 6: While many plots shared on \#PlotterTwitter are abstract, \#PlotterTwitter plots can be created from existing images or objects, such as photographs, paintings, and 3D scans. These plots were made by @PhotoPuck, @tasty_plots, @ajpiano, @yaronski, and @liviopacifico.

Paul Rickards

@paulrickards

Hey \#plottertwitter! I'd like to send you a postcard docs.google.com/forms/d/e/1FAl...

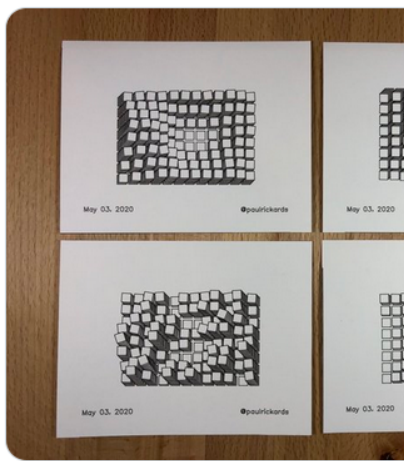

6:38 PM - May 5, $2020 \cdot$ Twitter for iPhone
Julien Gachadoat

@v3ga

It took some time but we'll, it finally arrived! Thanks

@paulrickards \#plottertwitter
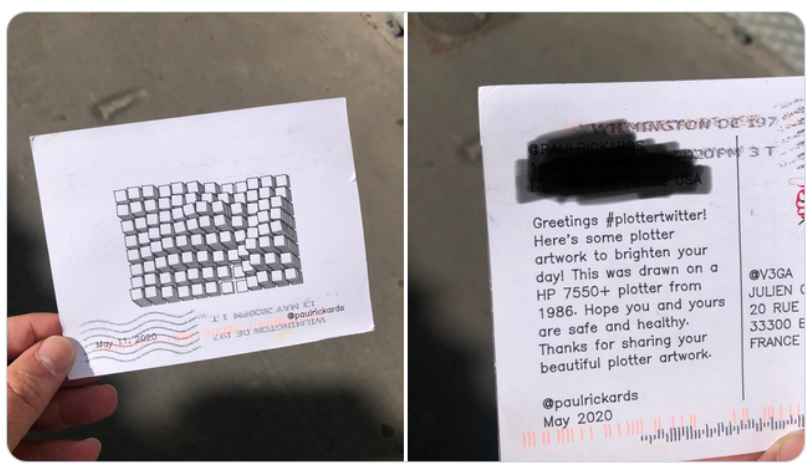

10:04 AM - Jul 11, 2020 - Twitter for iPhone

Figure 7: \#PlotterTwitter postcards sent by @ paulrickards show that the \#PlotterTwitter relationship extends beyond Twitter as physical plots are shared around the world. Postcard text: Greetings \#PlotterTwitter! Here's some plotter artwork to brighten your day! This was drawn on an HP 7550+ plotter from 1986. Hope you and yours are safe and healthy. Thanks for sharing your beautiful plotter artwork. @paulrickards May 2020

Further, we observed questions about materials to use with the plotter: which pens are refillable, how to properly anchor paper, how to achieve a certain effect with ink, or how to prevent a pen nib from wearing out. These questions represent a tiny selection of the topics addressed on \#PlotterTwitter. Altogether, the community has experimented with many configurations and serves as a reliable pool of crowdsourced information for those who are experimenting with novel plotter workflows.

\subsection{Foregrounding the Machine as a Co-Creator}

Instead of sharing finished artwork alone, \#PlotterTwitter members frequently portrayed the means by which they created their plots. 
We observed many tweets which emphasized the creator's relationship with their machine and the journey that they took to arrive together at a finished product. Some people prioritized discussing the machine's actions rather than the end goal, like @265design: "Took me a long time to set this one up but the process was therapeutic and the results pleasing." @qrs states, "It's very relaxing to watch the polargraph smoothly plot the [Lorenz] Attractor on the wall over several hours" while including an image of a hanging plotter gradually covering a large wall with curved lines. Instead of simply sharing the plotted work by itself, it was often pictured still sitting on the bed of the plotter, or even half-finished in an "action" shot mid-plot. These tweets highlight the importance of the machine as a collaborator in the artistic process, in stark contrast to the canonical workflow's notion of a machine as an obedient translator of digital formats to physical action.

Given the prominent role of the machine coupled with a responsive community, members were likely to share their experiences and struggles in working with the plotter itself. Specifically, defects or issues were often framed as serendipitous rather than problematic or undesirable. In one tweet, @artflychris described the defects in their plot as adding "originality and authenticity." Later, they tweet "Tried plotting a hatch version of a geometric portrait I made. Not entirely happy with it, but what I do love are the little ink defects - I could get lost in them! I heart them defects!" @revdancatt goes further, noting "When your scrap paper plotting tests start to look better than your real plots. Pure alchemy going on here. Now I want to see other peoples' rejected mis-plots." This spawned a new hashtag within the community: \#MisPlotWeekend. In the canonical workflow, errors in the final product are seen as requiring a repetition of the entire pipeline to address a mistake in one part of the whole. However, these tweets suggest that community members often celebrate the holistic process, including unintended behavior or "fails," as opportunities to generate new artworks, improve existing tools, and further explore the realm of possibility.

\subsection{Lightweight and Flexible Design Tools are Preferred over a Monolithic Approach to CAD}

While CAD was one of our more inclusive codes, with 236 instances, we found that tweets coded as CAD were very diverse in what they described. In other words, there is a large ecosystem of software used to create and manipulate digital files for drawing, and the diversity of this ecosystem defies a monolithic description. We identified three types of tools used to create and process digital designs: direct specification tools, parametric tools, and translational tools.

With direct specification tools, a user directly describes the geometry of an object and must manually create paths for the plotter to follow. Examples of these tools include vector graphics programs like Adobe Illustrator or Inkscape. The low-level and one-off nature of direct specification tools contrasts with parametric tools, where the latter focuses on creating a system of rules to generate paths. These rules can be adjusted and rewritten to quickly explore a wide space of designs, and they allow for exploration of emergent properties and randomness in a design system. More importantly, parametric environments can easily be shared with and remixed by other users. Some examples of these tools include conventional software like Grasshopper and Fusion360, but on \#PlotterTwitter, homespun, community-created web tools dominate. On \#PlotterTwitter, parametric tools often took the form of browser-based Javascript SVG generators such as @msurguy's "Flowlines" SVG generator. Encapsulated in a graphical web app, these parametric environments were easily shared through the \#PlotterTwitter community.

Translational tools convert between digital formats. For example, @msurguy's Squigglecam generates plottable lines from a raster image, @MAKIO135 developed a photo-hatching tool, and peakmap and city-road by @anvaka translate topographic and road map information into plottable SVGs. Further examples of communitycreated translational tools are displayed in Figure 6, where artists have processed paintings, 3D-scans, and photographs into plottable formats.

Many workflows displayed in our dataset used multiple tools from these categories, adopting SVG as an intermediary format to move between different design tools. This contrasts with the idea of a one-size-fits-all CAD tool-plotter users move fluidly between parametric tools and translational tools, often only using direct specification tools at the end to "clean up" drawings, or add modifications that are specific to an individual drawing. This suggests that community members might prefer a set of smaller specialized tools over a larger tool that offers less flexibility.

\subsection{Grappling with the Physical-Digital Divide}

\#PlotterTwitter community members constantly face a disconnect between digital tools and the physical properties of their machines and materials. Digital tools only show on-screen the path that the machine will eventually take, usually as a set of vector lines. However, the final result of a machine following a vector path depends on many factors, such as the acceleration and speed of the drawing instrument, the pressure, and the physical properties of the material. Vector files like SVGs still need to be modified to accommodate the eccentricities of different machines and materials. For example, a felt-tip pen may require higher speeds than a ballpoint pen to achieve a similar line width. Looking solely at the path on a screen is not enough confidently predict how a machine will perform.

People must manually resolve any undesirable disconnects between digital formats and plotted results, as few tools exist to support this process. To address this tension, they create custom calibration tools like swatch tests to understand how their plotter and materials will perform. They then use this information to make educated decisions about machine settings, such as which drawing implement to use or the speeds and pressures to draw with. @FogleBird demonstrates this in their process of drawing a bluebird, where they test various combinations of pen and pencil to create a dynamic range of grays in the final plot. When a community member asked about their process, @FogleBird revealed that they used the physical pencil shades in a swatch plot to make a final selection of pencils. The swatches also helped them determine how much to weigh down some pencils for ideal shading performance, which they achieved via a rubber band. If someone received the digital file that @FogleBird used to create this plot, they would still 
have to repeat this calibration procedure for their own machine, materials, and desired outcome.

Other people on \#Plottertwitter noticed and adjusted to the physical properties of their machines. @0Framerate noticed that on the Axidraw plotter, the downward pressure of the pen varies at different locations on the paper. They write, "the \#axidraw downward pressure, as you'd expect, is noticeably greater with arm extended out. Makes a difference for sensitive pastels", showing a pastel plot with noticeably lighter sections. @revdancatt explored ways of creating rubbings or height maps of objects by placing them under the paper and using the plotter to draw over them in a controlled manner. One member remarked "That's lovely! I really like using physical stuff to distort the digital plot." Another commenter described this process as "using all the degrees of freedom". This goes to show how \#PlotterTwitter members are innovating not only with digital tools, but physical properties as well.

\subsection{Niche Exploration Eclipses Generic Workflows}

Much of the content on \#PlotterTwitter exists in a niche of its own. We were surprised by the specificity of the tasks some artists set out to accomplish. Instead of simply using a plotter to draw a vector file, we saw that artists had a vested interest in creating custom, open-ended infrastructure that supported their vision. Rather than working within the constraints of prescriptive tools that may assume a specific use-case or workflow, this approach allows for great flexibility. Critically, we noticed that this intense exploration of the functionality provided by task-specific, custom tools was often prioritized and even praised over generic approaches to plotting.

For example, @Andy_Makes developed a pipeline dedicated to converting Magic: the Gathering cards to a plottable format. In one tweet, they reflect on this process: "I've been having a lot of fun doing plotter prints of Magic: The Gathering cards. Part of what makes it so compelling is programming my tools as I use them." With this reflection, they also share a time lapse video of their workflow where they can be seen working via code and a visual interface side-by-side. Later, they shared a screenshot of their tool (Figure 9), saying "Since every piece of card art has a different style, I didn't think a one-size-fits-all solution made sense. So I tried to give myself a suite of image processor techniques and a simple layering system to control them.”. While the tool is specific to Magic: the Gathering cards, it allows an encapsulated set of rules to be reused, extended, and shared. @Andy_Makes demonstrates that artists are not simply creating their own tools out of necessity; instead, they take pride in the development of environments that help them explore their own, specific niches. In this way, the infrastructure artists build is tightly interwoven with their automation workflows, ultimately existing alongside a finished plotted work as a creative artifact.

Keeping with the theme of open-ended exploration, we observed community members gravitating toward existing creative coding environments such as Processing, P5.js, or Turtletoy. R was also a popular choice, particularly for people who wanted to work with statistical or geographical libraries. In particular, @MikeMahoney218 used topographical libraries in RStats to translate topographical data into a dotted, plottable height map. In this case, they worked with the rayshader package to isolate, clean, and process the data and create a rendered image of a small mountain range. This involved using techniques and libraries specific to the $\mathrm{R}$ language. @MikeMahoney218 also needed to modify their workflow to isolate only parts of the mountain range above a certain height to plot. Viewed holistically, much of this workflow comprises processing different forms of data, from statistical to topographic, with plotting happening only after many stages of transforming data representations.

Other niche workflows on \#PlotterTwitter incorporate additional machines. @0Framerate, who we mentioned above for noticing variable pen pressure in their Axidraw plotter, followed another line of inquiry involving an embroidery machine. Before they had access to the machine, they used a plotter to visualize what the eventual embroidered stitches would look like by plotting a white gel pen on canvas. They demonstrated extensive experimentation during this time; for example, they shared that their plots needed to consist of one single line to mimic the continuous thread requirement of embroidered designs. They also documented the process of dialing in the pen's speed to achieve a realistic "bleed" effect onto the cloth that mimics the look of thread. Eventually, @0Framerate gained access to an embroidering machine and used it to actually embroider the designs they tested with the Axidraw. By simulating embroidery with the plotter, they were able to identify and address concerns before they had access to the machine.

These examples demonstrate community members' drive to develop wholly new workflows that integrate a variety of artistic mediums and existing technologies, all while continuing to promote creative, open-ended exploration. Rather than viewing these niche explorations as anomalies in a digital fabrication workflow, we might better understand them by framing them as innovations that are constantly expanding the total space of remixable workflows.

\section{DISCUSSION}

\subsection{Workflows Themselves Become the Medium}

Altogether, we understand the diversity of \#PlotterTwitter to be evidence of a pluralistic, community-driven approach to using digital fabrication tools alongside other software. Far from the standardizable canonical workflow designed for CNC in the 1950s, the breadth of work on \#PlotterTwitter constantly engages with new materials, software, machines, and ways of stringing them all together. We see that many cutting-edge uses of plotters come from people venturing into unknown territory with the help of the community. This approach differs from the notion of following "best practices" common in engineering, and suggests that we might better understand workflows-not just end products-as creative explorations that use computation as just one of a number of mediums.

In his book that explored how notions of craft carry over to digital forms, Malcolm McCullough writes that "the ability to explore a continuum of design spaces represents a fundamental advance toward using the computer as a medium [of craft]" [41, p. 172]. He adds, "tightening the loop between conception [of designs] and [machine] execution has the potential to reconcile some of the previous separation of design and fabrication that industrialization had previously imposed on craft" [p. 178]. In the case 

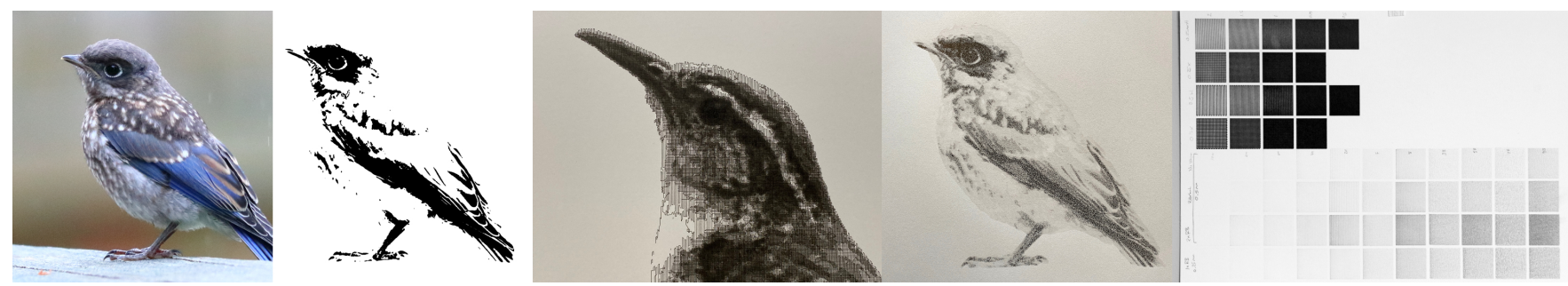

Figure 8: Creating a plot often requires people to understand how their tools will perform. @FogleBird shared their process of plotting a bluebird. They created a palette of grays by drawing squares with different pencils hardnesses and combinations of pencil and pen, and used it to determine which to use for the final plot. They also shared how they created the plottable image, which involved thresholding the photograph at different values.

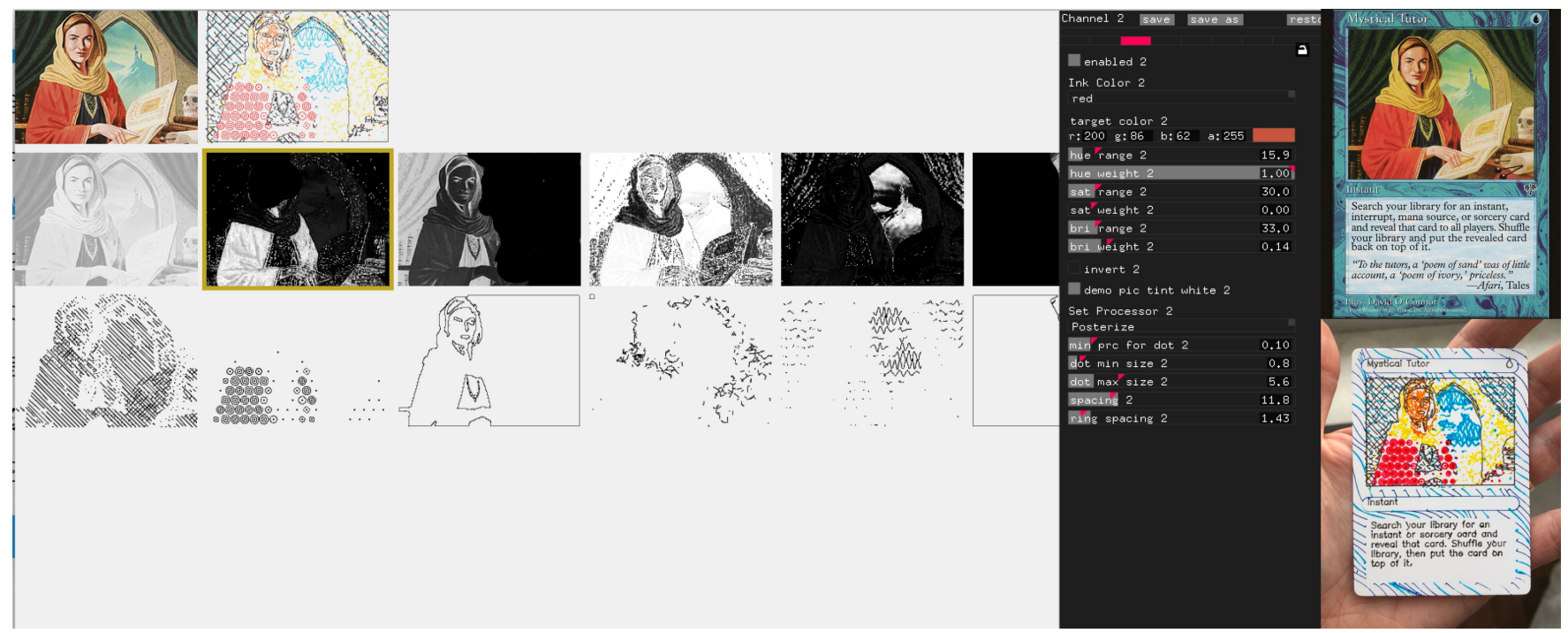

Figure 9: Artists on \#PlotterTwitter are constantly developing tools to support their specific workflow and vision. @Andy_Makes shared a screenshot of a custom tool they made in OpenFrameworks to convert a Magic: the Gathering card (top right) to a plottable format (bottom right).

of \#PlotterTwitter, a "continuum of design spaces" refers to not only different settings of a parametric model, but also to different spaces afforded by different software tools, different machines, different physical materials, and the different ways the maker weaves them together. To create truly original artwork with a plotter, community members must learn how to combine disparate tools and understand their limits while still maintaining an artistic vision. In addition to finished products, these creative struggles result in original workflows that illuminate compelling ways humans work with machines.

\section{2 \#PlotterTwitter Defies Norms of Who Engineers and How}

We view \#PlotterTwitter as more than a creative exploration of digital-machine infrastructure. Instead, it is a radical alternative to understanding $\mathrm{CNC}$ production against its historical roots, who produces, and how. Examining the relationship in architecture between $\mathrm{CAD}$ and politics, Cardoso Llach writes that "software systems and numerically controlled machines are not merely "instruments," or "tools," but rather versatile metaphors reconfiguring conceptions of design, materiality, work, and what it means to be creative" [10]. Others argue that CAD and associated tools, far from being neutral representations, actively negotiate power between the designer and other stakeholders [17, 23, 59].

The canonical workflow can exclude the perspectives of those who are not familiar with CNC processes or CAD tools. This is further reinforced by proprietary design software licenses that sometimes cost thousands of dollars, placing them firmly out of reach for a casual user. On \#PlotterTwitter, we see people engaging with $\mathrm{CNC}$ tools in a starkly different setting, against a community backdrop that looks fundamentally different than the pre-envisioned expert CAD userbase. Because no set of tools is explicitly assumed (and open source projects are actively promoted), exploration, rather than prescription, becomes the norm. The breadth of resources ensures that no single tool creator monopolizes others' creative capabilities. Community members become creators and maintainers of infrastructure and the realm of possibility expands alongside 
their skills and imagination. Beyond being passive consumers of fabrication tools, they are actively rewriting digital and machine infrastructure as a part of the creative process.

\subsection{Implications for HCI Research on Digital Fabrication}

Highlighting the plurality and radical nature of \#PlotterTwitter only makes sense against the historical backdrop of digital fabrication infrastructure, which prioritizes conformity. Yet, much of the creativity expressed in tweets from \#PlotterTwitter comes precisely from negotiating the constraints and mismatches of plotters to their digital representations. While the SVG file format does not support most machine-level instructions, community members have already contributed tips, tricks, and hacks to navigate this boundary. Researchers and practitioners who seek to contribute to the space of tools may best contribute by respecting the artistic goals that led to these hacks. This leads us to an important concession: we, as researchers and/or tool-builders, should not assume that we understand how people use digital fabrication technology. Further, we should not prescribe that makers use digital fabrication tools "the right way." We should instead leave room for, and perhaps even encourage, niche and case-specific interpretations.

The canonical workflow, which frames the use of CNC machinery as unidirectional and user-centered, is often useful for helping newcomers understand a basic framework of fabrication tools. However, as we have come to understand \#PlotterTwitter as an assemblage of evolving practices, using the canonical workflow to theorize how people use machines hides the myriad ways in which online community members are pushing the boundaries of computational craft. While developing more standards to better capture the space of novel workflows might seem attractive, we argue that researchers and practitioners should let go of the need to standardize, and instead adopt the current workflows of whomever they are working with. Deep-seated standards often result in hindrances to practitioners who are expanding the space of workflows by exploring the possibilities of expression with machines and software tools. We have already discussed such issues with the SVG file format, and this extends to the case of the STL file format for 3D printing, where, as Louis-Rosenberg writes, "file formats for additive manufacturing are lagging behind the capabilities of 3-D printing technology itself, and no one is doing anything about it" [39]. Software and their associated file formats should expose a continuum of design states. The use of entrenched standards is prone to fixing one set of priorities over new ones, just as SVGs fare well for vector graphics but not for machine toolpaths. As a result, HCI researchers studying fabrication should avoid developing prescriptive pipelines for makers, instead affording makers flexibility to choose between software and data representations that suit their current needs.

\section{CONCLUSION}

In direct opposition to the canonical workflow, \#PlotterTwitter members create, use, and maintain open software-machine environments. We see this as direct evidence that post-anthropocentric making is already taking place at the community level. However, research in digital fabrication within HCI largely exclusively espouses the anthropocentric view of making. Indeed, the very notion of personal fabrication that motivates work in the field inherently assumes that machines and their software are passive objects which faithfully obey the "person" without regard to material constraints. While this anthropocentric view is often useful, we risk limiting our understanding of the creative possibilities of digital fabrication if we do not also adopt the post-anthropocentric view. With \#PlotterTwitter, members strive to engage with digital and physical materials at the lowest level of machine control and to share their process with others. This refutes a fundamental vision of personal fabrication that Gershenfeld formulates as being "the ability to turn data into things and things into data" [20]. Instead of becoming one and other, things and data gradually achieve new forms in tandem within a complex network of interwoven processes that individuals explore, extend, and share. We call on HCI researchers to build and engage with maker communities through a post-anthropocentric view: an understanding that digital fabrication can be more than a series of steps, that materials have agency alongside a user's goals, and that opportunities for creative exploration are more important than seamless control.

\section{ACKNOWLEDGMENTS}

We would like to thank the 29 \#PlotterTwitter artists whose work appeared in this paper: @ManiacalLabs, @PhotoPuck, @artflychris, @liviopacifico, @ajpiano, @MAKIO135, @fastness, @jcupitt65, @0Framerate, @revdancatt, @265design, @v3ga, @Andy_Makes, @anvaka, @clarkkampfe, @FogleBird, @MikeMahoney218, @msurguy, @paulrickards, @qrs, @thomasp85, @cicerothedoge, @yaronski, @tasty_plots, @NadiehBremer, @kenjirototsuji, @lwrkalia, @mhkingsbury, and @plotslol. Thanks to Benjamin Mako Hill for feedback on early versions of this paper, and thanks to our reviewers for their thoughtful suggestions. This research was funded in part by the NSF IIS Human-Centered Computing program (\#2007045).

\section{REFERENCES}

[1] Lea Albaugh, Scott E. Hudson, Lining Yao, and Laura Devendorf. 2020. Investigating Underdetermination Through Interactive Computational Handweaving. In Proceedings of the 2020 ACM Designing Interactive Systems Conference (DIS '20). Association for Computing Machinery, New York, NY, USA, 1033-1046. https://doi.org/10.1145/3357236.3395538

[2] Celena Alcock, Nathaniel Hudson, and Parmit K. Chilana. 2016. Barriers to Using, Customizing, and Printing 3D Designs on Thingiverse. In Proceedings of the 19th International Conference on Supporting Group Work (GROUP '16). Association for Computing Machinery, New York, NY, USA, 195-199. https: //doi.org/10.1145/2957276.2957301

[3] Celena Alcock, Nathaniel Hudson, and Parmit K. Chilana. 2016. Barriers to Using, Customizing, and Printing 3D Designs on Thingiverse. In Proceedings of the 19th International Conference on Supporting Group Work (Sanibel Island, Florida, USA) (GROUP '16). Association for Computing Machinery, New York, NY, USA, 195-199. https://doi.org/10.1145/2957276.2957301

[4] Kristina Andersen, Ron Wakkary, Laura Devendorf, and Alex McLean. 2019. Digital Crafts-Machine-Ship: Creative Collaborations with Machines. Interactions 27, 1 (Dec. 2019), 30-35. https://doi.org/10.1145/3373644 Place: New York, NY, USA Publisher: Association for Computing Machinery.

[5] Cecilia R. Aragon, Sarah S. Poon, Andrés Monroy-Hernández, and Diana Aragon. 2009. A tale of two online communities: fostering collaboration and creativity in scientists and children. In Proceedings of the seventh ACM conference on Creativity and cognition (C\&amp; C '09). Association for Computing Machinery, New York, NY, USA, 9-18. https://doi.org/10.1145/1640233.1640239

[6] Jeffrey Bardzell, Shaowen Bardzell, and Austin Toombs. 2014. "now that's definitely a proper hack": self-made tools in hackerspaces. In Proceedings of the SIGCHI Conference on Human Factors in Computing Systems (CHI '14). Association for Computing Machinery, New York, NY, USA, 473-476. https: 
//doi.org/10.1145/2556288.2557221

[7] Patrick Baudisch, Arthur Silber, Yannis Kommana, Milan Gruner, Ludwig Wall Kevin Reuss, Lukas Heilman, Robert Kovacs, Daniel Rechlitz, and Thijs Roumen. 2019. Kyub: A 3D Editor for Modeling Sturdy Laser-Cut Objects. In Proceedings of the 2019 CHI Conference on Human Factors in Computing Systems (Glasgow, Scotland Uk) (CHI '19). Association for Computing Machinery, New York, NY, USA, 1-12. https://doi.org/10.1145/3290605.3300796

[8] G Blauvelt, $T$ Wrensch, and $M$ Eisenberg. 2000. Integrating craft materials and computation. Knowledge-Based Systems 13, 7 (Dec. 2000), 471-478. https: //doi.org/10.1016/S0950-7051(00)00063-0

[9] Amy Bruckman, Kurt Luther, and Casey Fiesler. 2015. When should we use real names in published accounts of internet research.

[10] Daniel Cardoso Llach. 2012. Builders of the vision : technology and the imagination of design. Thesis. Massachusetts Institute of Technology. https://dspace.mit.edu/ handle/1721.1/77775 Accepted: 2013-03-13T15:45:29Z.

[11] Zekun Chang, Tung D. Ta, Koya Narumi, Heeju Kim, Fuminori Okuya, Dongchi Li, Kunihiro Kato, Jie Qi, Yoshinobu Miyamoto, Kazuya Saito, and Yoshihiro Kawahara. 2020. Kirigami Haptic Swatches: Design Methods for Cut-and-Fold Haptic Feedback Mechanisms. In Proceedings of the 2020 CHI Conference on Human Factors in Computing Systems (CHI '20). Association for Computing Machinery, New York, NY, USA, 1-12. https://doi.org/10.1145/3313831.3376655

[12] Laura Devendorf, Katya Arquilla, Sandra Wirtanen, Allison Anderson, and Steven Frost. 2020. Craftspeople as Technical Collaborators: Lessons Learned through an Experimental Weaving Residency. In Proceedings of the 2020 CHI Conference on Human Factors in Computing Systems (CHI '20). Association for Computing Machinery, New York, NY, USA, 1-13. https://doi.org/10.1145/3313831.3376820 event-place: Honolulu, HI, USA.

[13] Laura Devendorf, Abigail De Kosnik, Kate Mattingly, and Kimiko Ryokai. 2016. Probing the Potential of Post-Anthropocentric 3D Printing. In Proceedings of the 2016 ACM Conference on Designing Interactive Systems (DIS '16). ACM, New York, NY, USA, 170-181. https://doi.org/10.1145/2901790.2901879

[14] Tamara Anna Efrat, Moran Mizrahi, and Amit Zoran. 2016. The Hybrid Bricolage: Bridging Parametric Design with Craft through Algorithmic Modularity. In Proceedings of the 2016 CHI Conference on Human Factors in Computing Systems (CHI '16). Association for Computing Machinery, New York, NY, USA, 5984-5995. https://doi.org/10.1145/2858036.2858441

[15] Madeleine Clare Elish. 2018. The stakes of uncertainty: Developing and integrating machine learning in clinical care. In Ethnographic Praxis in Industry Conference Proceedings, Vol. 2018. Wiley Online Library, 364-380.

[16] Sarah Evans, Katie Davis, Abigail Evans, Julie Ann Campbell, David P. Randall, Kodlee Yin, and Cecilia Aragon. 2017. More Than Peer Production: Fanfiction Communities as Sites of Distributed Mentoring. In Proceedings of the 2017 ACM Conference on Computer Supported Cooperative Work and Social Computing (CSCW '17). Association for Computing Machinery, New York, NY, USA, 259-272. https: //doi.org/10.1145/2998181.2998342

[17] Boris Ewenstein and Jennifer Whyte. 2009. Knowledge Practices in Design: The Role of Visual Representations as 'Epistemic Objects'. Organization Studies 30, 1 (Jan. 2009), 07-30. https://doi.org/10.1177/0170840608083014

[18] Raune Frankjær and Peter Dalsgaard. 2018. Understanding Craft-Based Inquiry in HCI. In Proceedings of the 2018 Designing Interactive Systems Conference (DIS '18). Association for Computing Machinery, New York, NY, USA, 473-484. https: //doi.org/10.1145/3196709.3196750 event-place: Hong Kong, China.

[19] Clifford Geertz. 1973. The interpretation of cultures: selected essays. Basic Books, New York.

[20] Neil Gershenfeld. 2015. How to Make Almost Anything. https://www. foreignaffairs.com/articles/2012-09-27/how-make-almost-anything

[21] Mary L. Gray and Siddharth Suri. 2019. Ghost Work: How to Stop Silicon Valley from Building a New Global Underclass. HMH Books.

[22] Shiqing He and Eytan Adar. 2020. Plotting with Thread: Fabricating Delicate Punch Needle Embroidery with X-Y Plotters. In Proceedings of the 2020 ACM Designing Interactive Systems Conference (DIS '20). Association for Computing Machinery, New York, NY, USA, 1047-1057. https://doi.org/10.1145/3357236. 3395540 event-place: Eindhoven, Netherlands.

[23] Kathryn Henderson. 1991. Flexible Sketches and Inflexible Data Bases: Visual Communication, Conscription Devices, and Boundary Objects in Design Engineering. Science, Technology, \& Human Values 16, 4 (Oct. 1991), 448-473. https://doi.org/10.1177/016224399101600402

[24] Benjamin Mako Hill and Andrés Monroy-Hernández. 2013. The cost of collaboration for code and art: evidence from a remixing community. In Proceedings of the 2013 conference on Computer supported cooperative work (CSCW '13). Association for Computing Machinery, New York, NY, USA, 1035-1046. https://doi.org/10.1145/2441776.2441893

[25] Megan Hofmann, Gabriella Hann, Scott E. Hudson, and Jennifer Mankoff. 2018 Greater than the Sum of Its PARTs: Expressing and Reusing Design Intent in 3D Models. In Proceedings of the 2018 CHI Conference on Human Factors in Computing Systems (Montreal QC, Canada) (CHI '18). Association for Computing Machinery, New York, NY, USA, 1-12. https://doi.org/10.1145/3173574.3173875
[26] Nathaniel Hudson, Celena Alcock, and Parmit K. Chilana. 2016. Understanding Newcomers to 3D Printing: Motivations, Workflows, and Barriers of Casual Makers. In Proceedings of the 2016 CHI Conference on Human Factors in Computing Systems (CHI '16). Association for Computing Machinery, New York, NY, USA, 384-396. https://doi.org/10.1145/2858036.2858266

[27] Tim Ingold. 2013. Making: anthropology, archaeology, art and architecture. Routledge, London ; New York.

[28] Lilly Irani. 2019. Chasing Innovation: Making Entrepreneurial Citizens in Modern India. Princeton University Press.

[29] Jennifer Jacobs, Joel Brandt, Radomír Mech, and Mitchel Resnick. 2018. Extending Manual Drawing Practices with Artist-Centric Programming Tools. In Proceedings of the 2018 CHI Conference on Human Factors in Computing Systems (CHI '18). Association for Computing Machinery, New York, NY, USA, 1-13. https://doi. org $/ 10.1145 / 3173574.3174164$

[30] Jennifer Jacobs, David Mellis, Amit Zoran, Cesar Torres, Joel Brandt, and Theresa Jean Tanenbaum. 2016. Digital Craftsmanship: HCI Takes on Technology as an Expressive Medium. In Proceedings of the 2016 ACM Conference Companion Publication on Designing Interactive Systems (DIS '16 Companion). Association for Computing Machinery, New York, NY, USA, 57-60. https://doi.org/10.1145/2908805.2913018

[31] Jennifer Jacobs and Amit Zoran. 2015. Hybrid Practice in the Kalahari: Design Collaboration through Digital Tools and Hunter-Gatherer Craft. In Proceedings of the 33rd Annual ACM Conference on Human Factors in Computing Systems (CHI '15). Association for Computing Machinery, New York, NY, USA, 619-628. https://doi.org/10.1145/2702123.2702362 event-place: Seoul, Republic of Korea.

[32] Joy Kim, Maneesh Agrawala, and Michael S. Bernstein. 2017. Mosaic: Designing Online Creative Communities for Sharing Works-in-Progress. In Proceedings of the 2017 ACM Conference on Computer Supported Cooperative Work and Social Computing. ACM, New York, NY, USA, 246-258. https://doi.org/10.1145/2998181. 2998195

[33] Karin D Knorr. 1979. Tinkering Toward Success: Prelude to a Theory of Scientific Practice'. Theory and Society 8 (1979), 347-76.

[34] Robert Kovacs, Anna Seufert, Ludwig Wall, Hsiang-Ting Chen, Florian Meinel, Willi Müller, Sijing You, Maximilian Brehm, Jonathan Striebel, Yannis Kommana, Alexander Popiak, Thomas Bläsius, and Patrick Baudisch. 2017. TrussFab: Fabricating Sturdy Large-Scale Structures on Desktop 3D Printers. In Proceedings of the 2017 CHI Conference on Human Factors in Computing Systems (CHI '17). Association for Computing Machinery, New York, NY, USA, 2606-2616. https://doi.org/10.1145/3025453.3026016

[35] Stacey Kuznetsov and Eric Paulos. 2010. Rise of the expert amateur: DIY projects, communities, and cultures. In Proceedings of the 6th Nordic Conference on Human-Computer Interaction: Extending Boundaries (NordiCHI '10). Association for Computing Machinery, New York, NY, USA, 295-304. https: //doi.org/10.1145/1868914.1868950

[36] Bruno Latour and Steve Woolgar. 1979. Laboratory Life: The Construction of Scientific Facts. Princeton University Press.

[37] Danny Leen, Nadya Peek, and Raf Ramakers. 2020. LamiFold: Fabricating Objects with Integrated Mechanisms Using a Laser Cutter Lamination Workflow. In Proceedings of the 33rd Annual ACM Symposium on User Interface Software and Technology (Virtual Event, USA) (UIST'20). Association for Computing Machinery, New York, NY, USA, 304-316. https://doi.org/10.1145/3379337.3415885

[38] Silvia M. Lindtner. 2020. Prototype Nation: China and the Contested Promise of Innovation. Princeton University Press.

[39] Jesse Louis-Rosenberg. 2016. Drowning in triangle soup: the quest for a better 3-D printing file format. XRDS: Crossroads, The ACM Magazine for Students 22, 3 (April 2016), 58-62. https://doi.org/10.1145/2893503

[40] Li-Ke Ma, Yizhonc Zhang, Yang Liu, Kun Zhou, and Xin Tong. 2017. Computational design and fabrication of soft pneumatic objects with desired deformations. ACM Transactions on Graphics 36, 6 (Nov. 2017), 239:1-239:12. https://doi.org/10.1145/3130800.3130850

[41] Malcolm McCullough. 1996. Abstracting Craft: The Practiced Digital Hand. MIT Press, Cambridge, MA, USA

[42] A. M. Mehta and D. Rus. 2014. An end-to-end system for designing mechanical structures for print-and-fold robots. In 2014 IEEE International Conference on Robotics and Automation (ICRA). Institute of Electrical and Electronics Engineers (IEEE), New York, NY, 1460-1465. https://doi.org/10.1109/ICRA.2014.6907044

[43] Michael Hansmeyer. 2010. Subdivided Columns.

[44] Annemarie Mol. 2002. The Body Multiple: Ontology in Medical Practice. Duke University Press.

[45] Andrés Monroy-Hernández, Benjamin Mako Hill, Jazmin Gonzalez-Rivero, and danah boyd. 2011. Computers can't give credit: how automatic attribution falls short in an online remixing community. In Proceedings of the SIGCHI Conference on Human Factors in Computing Systems (CHI '11). Association for Computing Machinery, New York, NY, USA, 3421-3430. https://doi.org/10.1145/1978942. 1979452

[46] Vidya Narayanan, Lea Albaugh, Jessica Hodgins, Stelian Coros, and James Mccann. 2018. Automatic Machine Knitting of 3D Meshes. ACM Transactions on Graphics 37, 3 (Aug. 2018), 35:1-35:15. https://doi.org/10.1145/3186265 
[47] David F. Noble. 1984. Forces of production: a social history of industrial automation (1st ed ed.). Knopf, New York.

[48] Lora Oehlberg, Wesley Willett, and Wendy E. Mackay. 2015. Patterns of Physical Design Remixing in Online Maker Communities. In Proceedings of the 33rd Annual ACM Conference on Human Factors in Computing Systems (CHI '15). Association for Computing Machinery, New York, NY, USA, 639-648. https://doi.org/10. $1145 / 2702123.2702175$

[49] Hyunjoo Oh, Jeeeun Kim, Cory Morales, Mark Gross, Michael Eisenberg, and Sherry Hsi. 2017. FoldMecha: Exploratory Design and Engineering of Mechanical Papercraft. In Proceedings of the Eleventh International Conference on Tangible, Embedded, and Embodied Interaction (TEI '17). Association for Computing Machinery, New York, NY, USA, 131-139. https://doi.org/10.1145/3024969.3024991 event-place: Yokohama, Japan.

[50] openFrameworks. 2020. openFrameworks Forum. https://forum. openframeworks.cc/

[51] p5.js. 2020. p5.js 1.0 Contributors Zine. http://euro-travel-example.com/index. htm

[52] Tyler Pace, Austin Toombs, Shad Gross, Tony Pattin, Jeffrey Bardzell, and Shaowen Bardzell. 2013. A Tribute to Mad Skill: Expert Amateur Visuality and World of Warcraft Machinima. In Proceedings of the SIGCHI Conference on Human Factors in Computing Systems (Paris, France) (CHI '13). Association for Computing Machinery, New York, NY, USA, 2019-2028. https: //doi.org/10.1145/2470654.2466267

[53] Nadya Peek, James Coleman, Ilan Moyer, and Neil Gershenfeld. 2017. Cardboard Machine Kit: Modules for the Rapid Prototyping of Rapid Prototyping Machines In Proceedings of the 2017 CHI Conference on Human Factors in Computing Systems. ACM, Denver Colorado USA, 3657-3668. https://doi.org/10.1145/3025453. 3025491

[54] Processing. 2020. Processing Forum. https://discourse.processing.org/

[55] Jie Qi and Leah Buechley. 2012. Animating Paper Using Shape Memory Alloys. In Proceedings of the SIGCHI Conference on Human Factors in Computing Systems (CHI '12). Association for Computing Machinery, New York, NY, USA, 749-752. https://doi.org/10.1145/2207676.2207783 event-place: Austin, Texas, USA.

[56] Jie Qi, Asli Demir, and Joseph A. Paradiso. 2017. Code Collage: Tangible Programming On Paper With Circuit Stickers. In Proceedings of the 2017 CHI Conference Extended Abstracts on Human Factors in Computing Systems (CHI EA '17). Association for Computing Machinery, New York, NY, USA, 1970-1977. https://doi.org/10.1145/3027063.3053084 event-place: Denver, Colorado, USA.

[57] Andrew Quitmeyer and Hannah Perner-Wilson. 2015. Wearable Studio Practice Design Considerations for Digital Crafting in Harsh Environments. In Adjunct Proceedings of the 2015 ACM International foint Conference on Pervasive and Ubiquitous Computing and Proceedings of the 2015 ACM International Symposium on Wearable Computers (UbiComp/ISWC'15 Adjunct). Association for Computing Machinery, New York, NY, USA, 1285-1293. https://doi.org/10.1145/2800835. 2807926 event-place: Osaka, Japan.

[58] Raf Ramakers, Fraser Anderson, Tovi Grossman, and George Fitzmaurice. 2016 RetroFab: A Design Tool for Retrofitting Physical Interfaces using Actuators, Sensors and 3D Printing. In Proceedings of the 2016 CHI Conference on Human Factors in Computing Systems (CHI '16). Association for Computing Machinery, New York, NY, USA, 409-419. https://doi.org/10.1145/2858036.2858485

[59] Daniela Retelny and Pamela Hinds. 2016. Embedding Intentions in Drawings: How Architects Craft and Curate Drawings to Achieve Their Goals. In Proceedings of the 19th ACM Conference on Computer-Supported Cooperative Work \& Social Computing. ACM, New York, NY, USA, 1310-1322. https://doi.org/10.1145/ 2818048.2819932

[60] Richard Dupont. 2008. Terminal Stage.

[61] Alec Rivers, Ilan E. Moyer, and Frédo Durand. 2012. Position-Correcting Tools for 2D Digital Fabrication. ACM Trans. Graph. 31, 4, Article 88 (July 2012), 7 pages. https://doi.org/10.1145/2185520.2185584

[62] Isabelle Rivoal and Noel B. Salazar. 2013. Contemporary Ethnographic Practice and the Value of Serendipity. SSRN Scholarly Paper ID 2321082. Social Science Research Network, Rochester, NY. https://papers.ssrn.com/abstract=2321082

[63] S. Roosth. 2017. Synthetic: How Life Got Made. University of Chicago Press.

[64] Ricarose Roque, Natalie Rusk, and Mitchel Resnick. 2016. Supporting Diverse and Creative Collaboration in the Scratch Online Community. In Mass Collaboration and Education, Ulrike Cress, Johannes Moskaliuk, and Heisawn Jeong (Eds.). Springer International Publishing, Cham, 241-256. https://doi.org/10.1007/9783-319-13536-6_12

[65] Daniela K. Rosner. 2018. Critical fabulations: reworking the methods and margins of design. The MIT Press, Cambridge, MA.

[66] Roxy Paine. 2001. Drawing Machine.
[67] Hidekazu Saegusa, Thomas Tran, and Daniela K. Rosner. 2016. Mimetic Machines: Collaborative Interventions in Digital Fabrication with Arc. In Proceedings of the 2016 CHI Conference on Human Factors in Computing Systems (CHI '16). ACM, New York, NY, USA, 6008-6013. https://doi.org/10.1145/2858036.2858475

[68] Ryan Schmidt and Matt Ratto. 2013. Design-to-Fabricate: Maker Hardware Requires Maker Software. IEEE Computer Graphics and Applications 33, 6 (Nov. 2013), 26-34. https://doi.org/10.1109/MCG.2013.90 Conference Name: IEEE Computer Graphics and Applications.

[69] Adriana Schulz, Cynthia Sung, Andrew Spielberg, Wei Zhao, Robin Cheng, Eitan Grinspun, Daniela Rus, and Wojciech Matusik. 2017. Interactive robogami: An end-to-end system for design of robots with ground locomotion. The International Journal of Robotics Research 36, 10 (Sept. 2017), 1131-1147. https://doi.org/10. $1177 / 0278364917723465$

[70] Susanne Seitinger, Daniel M. Taub, and Alex S. Taylor. 2010. Light Bodies: Exploring Interactions with Responsive Lights. In Proceedings of the Fourth International Conference on Tangible, Embedded, and Embodied Interaction (TEI '10). ACM, New York, NY, USA, 113-120. https://doi.org/10.1145/1709886.1709908

[71] Richard Sennett. 2008. The craftsman. Yale Univ. Press, New Haven. OCLC: 263736322.

[72] Lucille Alice Suchman. 2007. Human-machine reconfigurations: plans and situated actions (2nd ed ed.). Cambridge University Press, Cambridge ; New York. OCLC: ocm64592145.

[73] Rundong Tian, Vedant Saran, Mareike Kritzler, Florian Michahelles, and Eric Paulos. 2019. Turn-by-Wire: Computationally Mediated Physical Fabrication. In Proceedings of the 32nd Annual ACM Symposium on User Interface Software and Technology (UIST '19). Association for Computing Machinery, New Orleans, LA, USA, 713-725. https://doi.org/10.1145/3332165.3347918

[74] Rundong Tian, Sarah Sterman, Ethan Chiou, Jeremy Warner, and Eric Paulos. 2018. MatchSticks: Woodworking through Improvisational Digital Fabrication. In Proceedings of the 2018 CHI Conference on Human Factors in Computing Systems. Association for Computing Machinery, New York, NY, USA, 1-12. http://doi. org $/ 10.1145 / 3173574.3173723$

[75] Cesar Torres, Jasper O'Leary, Molly Nicholas, and Eric Paulos. 2017. Illumination Aesthetics: Light As a Creative Material Within Computational Design. In Proceedings of the 2017 CHI Conference on Human Factors in Computing Systems (CHI '17). ACM, New York, NY, USA, 6111-6122. https://doi.org/10.1145/3025453.3025466

[76] Cesar Torres and Eric Paulos. 2015. MetaMorphe: Designing Expressive 3D Models for Digital Fabrication. In Proceedings of the 2015 ACM SIGCHI Conference on Creativity and Cognition (C\&C '15). ACM, New York, NY, USA, 73-82. https: //doi.org/10.1145/2757226.2757235

[77] Tiffany Tseng and Mitchel Resnick. 2014. Product Versus Process: Representing and Appropriating DIY Projects Online. In Proceedings of the 2014 Conference on Designing Interactive Systems (DIS '14). ACM, New York, NY, USA, 425-428. https://doi.org/10.1145/2598510.2598540

[78] Tom Valkeneers, Danny Leen, Daniel Ashbrook, and Raf Ramakers. 2019. StackMold: Rapid Prototyping of Functional Multi-Material Objects with Selective Levels of Surface Details. In Proceedings of the 32nd Annual ACM Symposium on User Interface Software and Technology (UIST '19). Association for Computing Machinery, New York, NY, USA, 687-699. https://doi.org/10.1145/3332165.3347915

[79] Ron Wakkary and Leah Maestri. 2007. The resourcefulness of everyday design. In Proceedings of the 6th ACM SIGCHI conference on Creativity \& cognition (C\&amp;C '07). Association for Computing Machinery, New York, NY, USA, 163-172. https: //doi.org/10.1145/1254960.1254984

[80] Karl D.D. Willis, Cheng Xu, Kuan-Ju Wu, Golan Levin, and Mark D. Gross. 2011. Interactive fabrication: new interfaces for digital fabrication. In Proceedings of the fifth international conference on Tangible, embedded, and embodied interaction - TEI '11. ACM Press, Funchal, Portugal, 69. https://doi.org/10.1145/1935701.1935716

[81] Nur Yildirim, James McCann, and John Zimmerman. 2020. Digital Fabrication Tools at Work: Probing Professionals' Current Needs and Desired Futures. In Proceedings of the 2020 CHI Conference on Human Factors in Computing Systems (Honolulu, HI, USA) (CHI '20). Association for Computing Machinery, New York, NY, USA, 1-13. https://doi.org/10.1145/3313831.3376621

[82] Clement Zheng, HyunJoo Oh, Laura Devendorf, and Ellen Yi-Luen Do. 2019. Sensing Kirigami. In Proceedings of the 2019 on Designing Interactive Systems Conference (DIS '19). Association for Computing Machinery, New York, NY, USA, 921-934. https://doi.org/10.1145/3322276.3323689 event-place: San Diego, CA, USA.

[83] Amit Zoran and Joseph A. Paradiso. 2013. FreeD: A Freehand Digital Sculpting Tool. In Proceedings of the SIGCHI Conference on Human Factors in Computing Systems (CHI '13). ACM, New York, NY, USA, 2613-2616. https://doi.org/10. $1145 / 2470654.2481361$ 